\title{
Mode-coupling instability in a single-layer complex plasma crystal: Strong damping regime
}

\author{
V. Nosenko, ${ }^{1,2, a)}$ S. K. Zhdanov, ${ }^{1}$ J. Carmona-Reyes, ${ }^{2}$ and T. W. Hyde ${ }^{2}$ \\ ${ }^{1)}$ Institut für Materialphysik im Weltraum, Deutsches Zentrum für Luft- und Raumfahrt (DLR), D-82234 Weßling, \\ Germany \\ ${ }^{2)}$ Center for Astrophysics, Space Physics, and Engineering Research (CASPER), Baylor University, Waco, \\ Texas 76798-7310, USA
}

(Dated: 12 September 2018)

Mode-coupling instability (MCI) in a single-layer complex plasma crystal was studied experimentally in the regime of strong neutral gas damping. To trigger MCI, the discharge power was reduced at constant gas pressure. Surprisingly, at the onset of MCI the mean interparticle spacing became larger and the normalized neutral gas damping rate marginally increased. A steady-state regime where MCI occurred but was suppressed by the neutral gas friction was observed. Suppressed MCI caused heating up of the plasma crystal but did not lead to its melting. A theoretical model is proposed which describes well our experimental observations.

PACS numbers: $52.27 . \mathrm{Lw}, 52.27 . \mathrm{Gr}, 52.35 . \mathrm{Fp}$

\section{INTRODUCTION}

Wave modes of many physical systems can become coupled to each other. Mode coupling can lead to new interesting effects such as the mode-coupling instability (MCI) which was predicted theoretically ${ }^{1}$ and observed experimentally in single-layer (two-dimensional, 2D) complex plasma crystals $^{2,3}$. Complex plasmas are suspensions of charged microparticles in a weakly ionized gas ${ }^{5}$. The particle subsystem is often strongly coupled and can possess a liquid or even crystalline order. Due to their unique properties (stretched spatial and temporal scales, easy observability), plasma crystals have been widely used as model systems to study various phenomena such as phase transitions $^{6-8}$, transport phenomena ${ }^{9-13}$, and waves ${ }^{14-17}$ in real time and at the level of individual particles. MCI can occur in a plasma crystal when the dispersion relations of the two dust-lattice wave modes, longitudinal in-plane and transverse out-of-plane intersect. In the vicinity of their intersection, a new hybrid mode appears which is unstable ${ }^{4}$. If not suppressed by the neutral gas friction, it will grow exponentially with time and can result in the crystal melting ${ }^{2,3}$.

Experimental observations of MCI reported in the literature were usually made at relatively low (normalized) neutral gas damping rate, up to $\nu / \omega_{0}=0.18$, see Table I in Ref. ${ }^{3}$ (here, $\nu$ is the Epstein damping rate ${ }^{18}$ and $\omega_{0}$ is a frequency scale of the $2 \mathrm{D}$ plasma crystal ${ }^{19}$ ). In a recent experiment $^{20}$, MCI was observed at a relatively high gas pressure of $12 \mathrm{~Pa}$, corresponding to $\nu / \omega_{0}=0.48$. Deep mode crossing and eventual melting of the plasma crystal were reported.

In the present work, we systematically study MCI at the gas pressures of $8-14 \mathrm{~Pa}$, corresponding to $\nu / \omega_{0}$ up to 0.51 . At the onset of MCI, a steady-state regime

\footnotetext{
a) Electronic mail: V.Nosenko@dlr.de
}

was observed where deep mode crossing occurred but the hybrid mode growth was suppressed by the neutral gas drag. Under these conditions, the plasma crystal was heated up by MCI, but did not melt. Such a regime was predicted theoretically and observed in a moleculardynamics simulation in Ref. ${ }^{3}$.

\section{EXPERIMENTAL METHOD}

The experiments reported in this paper were performed in CASPER's modified Gaseous Electronics Conference (GEC) rf reference cell ${ }^{20-22}$. Capacitively coupled discharge in argon was sustained by up to $40 \mathrm{~W}$ of $\mathrm{rf}$ power at $13.56 \mathrm{MHz}$. The lower (powered) disk-shaped electrode had a $1 \mathrm{~mm}$ deep, $25.4 \mathrm{~mm}$ diameter depression for radial particle confinement; a grounded ring-shaped electrode above it and the chamber walls served as the other electrode. Melamine-formaldehyde microspheres ${ }^{23}$ with a diameter of $8.9 \pm 0.09 \mu \mathrm{m}$ were suspended in the plasma sheath above the lower electrode. The particles formed a single-layer suspension where they arranged themselves in a triangular lattice with hexagonal symmetry. This plasma crystal was "purified" using the standard method ${ }^{24}$. The argon pressure $p_{\mathrm{Ar}}$ was then set to a value in the range of $p_{\mathrm{Ar}}=8-17 \mathrm{~Pa}$ and the forward discharge power $P_{\mathrm{rf}}$ was stepwise reduced from 40 to $1 \mathrm{~W}$. The ensuing weakening of the vertical confinement for particles ${ }^{2}$ resulted in eventual departure from the single-layer structure of the particle suspension. In the present paper, we focused on the very first stages of this process. The same particle suspension was used for all combinations of $p_{\mathrm{Ar}}$ and $P_{\mathrm{rf}}$.

The particle suspension was illuminated by two (red diode) laser sheets, horizontal and vertical, and imaged from the top and from the side by two Photron FASTCAM PCI 1024 cameras operating at 125 frames per second. The cameras were equipped with K-2 Infinity lenses (with a focal length of about $30 \mathrm{~cm}$ ) and bandpass inter- 
ference filters corresponding to the lasers' wavelengths. For the side view, we selected a vertical slice which contained the feature of interest, e.g. a crystal area melted by MCI. The particles were then identified and traced from frame to frame using a method where the particle image intensity profiles were analyzed and their first moments taken as the particle coordinates ${ }^{25}$.

The particle charge $Q$ and screening parameter $\kappa \equiv$ $a / \lambda$ were calculated using the method of Ref. ${ }^{26}$ (see also Section IV C). The mean interparticle spacing $a$ was measured as the position of the first peak in the pair correlation function for particles $g(r)$. The static structure factor of the particle suspension was calculated as $S(\mathbf{k})=N^{-1}\left\langle\sum_{l, m} e^{i \mathbf{k} \cdot\left(\mathbf{r}_{l}-\mathbf{r}_{m}\right)}\right\rangle$, where $N$ is the number of particles, $\mathbf{r}$ is the particle position, the sum runs over all pairs of particles $l$ and $m$, and the averaging is performed over time.

The fluctuation spectra of the particle velocity for the longitudinal in-plane and out-of-plane modes were calculated from the top-view and side-view particle video, respectively, using a method based on the Fourier transform in the space and time domains of the binned particle velocity data ${ }^{20,26}$. To reveal the angular dependence of the particle velocity spectra, we integrated them over the hot-spot frequency and plotted the resulting maps in the wave vector $\mathbf{k}$ plane, using the method of Ref. ${ }^{3}$. The wave propagation direction $\theta$ was measured in the crystal plane relative to the direction from a particle to the midpoint of its two nearest neighbors.

\section{RESULTS}

The first response of the particle suspension to weakening vertical confinement was the appearance of spinning vertical pairs of particles (torsions) at the gas pressures of $p_{\mathrm{Ar}}=14 \mathrm{~Pa}$ and higher, and MCI at the pressures of $12 \mathrm{~Pa}$ and lower. This corroborates the observation of MCI at $12 \mathrm{~Pa}$ in a similar experiment in Ref. ${ }^{20}$. In the experimental run with $p_{\mathrm{Ar}}=14 \mathrm{~Pa}$, MCI started when a single torsion was already present in the lattice. The results of the experimental runs with $p_{\mathrm{Ar}}=8-14 \mathrm{~Pa}$ are summarized in Table I. MCI was consistently observed at high normalized damping rates $\nu / \omega_{0}$ up to 0.51. Analysis of data in Table I reveals two interesting and counterintuitive trends. First, at the onset of MCI the mean interparticle spacing $a$ becomes larger. This trend is clearly seen in Fig. 1(a), where the evolution of the plasma crystal parameters in the experimental run with $p_{\mathrm{Ar}}=12 \mathrm{~Pa}$ is shown. This observation apparently contradicts the requirement that the plasma crystal density exceed a threshold for the MCI to start ${ }^{3}$. Second, the normalized neutral gas damping rate $\nu / \omega_{0}$ does not change much or may even become marginally larger, when MCI starts, see Fig. 1(b) and Table I.

These apparent contradictions with the requirements of the confinement and damping thresholds are explained by the fact that the experimental control parameters $\left(p_{\mathrm{Ar}}\right.$
TABLE I. Parameters of the single-layer plasma crystal in different experimental runs. Shown are the pressure of argon $p_{\mathrm{Ar}}, \mathrm{rf}$ discharge forward power $P_{\mathrm{rf}}$, particle charge $Q$, mean interparticle spacing $a$, screening length $\lambda$, screening parameter $\kappa \equiv a / \lambda$, neutral gas damping rate $\nu, 2 \mathrm{D}$ frequency scale $\omega_{0}$, and normalized neutral gas damping rate $\nu / \omega_{0}$. The error bars are $\pm 10 \%$ for $|Q|, \pm 2 \%$ for $a$, and $\pm 15 \%$ for $\kappa$ and $\lambda$. The last column indicates whether MCI occurred in that experimental run.

\begin{tabular}{ccccccccccc}
\hline \hline & $\begin{array}{c}p_{\mathrm{Ar}} \\
(\mathrm{Pa})\end{array}$ & $\begin{array}{c}P_{\mathrm{rf}} \\
(\mathrm{W})\end{array}$ & $\begin{array}{c}|Q| \\
(e)\end{array}$ & $\begin{array}{c}a \\
(\mu \mathrm{m})\end{array}$ & $\begin{array}{c}\lambda \\
(\mu \mathrm{m})\end{array}$ & $\begin{array}{c}\nu \\
\left(\mathrm{s}^{-1}\right)\end{array}$ & $\begin{array}{c}\omega_{0} \\
\left(\mathrm{~s}^{-1}\right)\end{array}$ & $\nu / \omega_{0}$ & $\mathrm{MCI}$ \\
\hline 1 & 8 & 30 & 10560 & 229 & 130 & 1.78 & 9.5 & 62.0 & 0.15 & No \\
2 & 8 & 25 & 8640 & 240 & 340 & 0.70 & 9.5 & 47.3 & 0.20 & Yes \\
\hline 3 & 10.1 & 22 & 8590 & 240 & 230 & 1.03 & 12.1 & 47.0 & 0.26 & No \\
4 & 10.1 & 16 & 9240 & 260 & 310 & 0.84 & 12.1 & 44.9 & 0.27 & Yes \\
\hline 5 & 12 & 22 & 8030 & 242 & 200 & 1.20 & 14.3 & 43.4 & 0.33 & No \\
6 & 12 & 15 & 9160 & 258 & 250 & 1.05 & 14.3 & 45.0 & 0.32 & No \\
7 & 12 & 11.5 & 9210 & 271 & 360 & 0.76 & 14.3 & 42.0 & 0.34 & Yes \\
8 & 12 & 11 & 9110 & 275 & 350 & 0.78 & 14.3 & 40.7 & 0.35 & Yes \\
\hline 9 & 14 & 22 & 8360 & 253 & 180 & 1.40 & 16.7 & 42.3 & 0.39 & No \\
10 & 14 & 8 & 9770 & 299 & 330 & 0.92 & 16.7 & 38.5 & 0.43 & No \\
11 & 14 & 6 & 8720 & 310 & 340 & 0.92 & 16.7 & 32.5 & 0.51 & Yes \\
\hline \hline
\end{tabular}
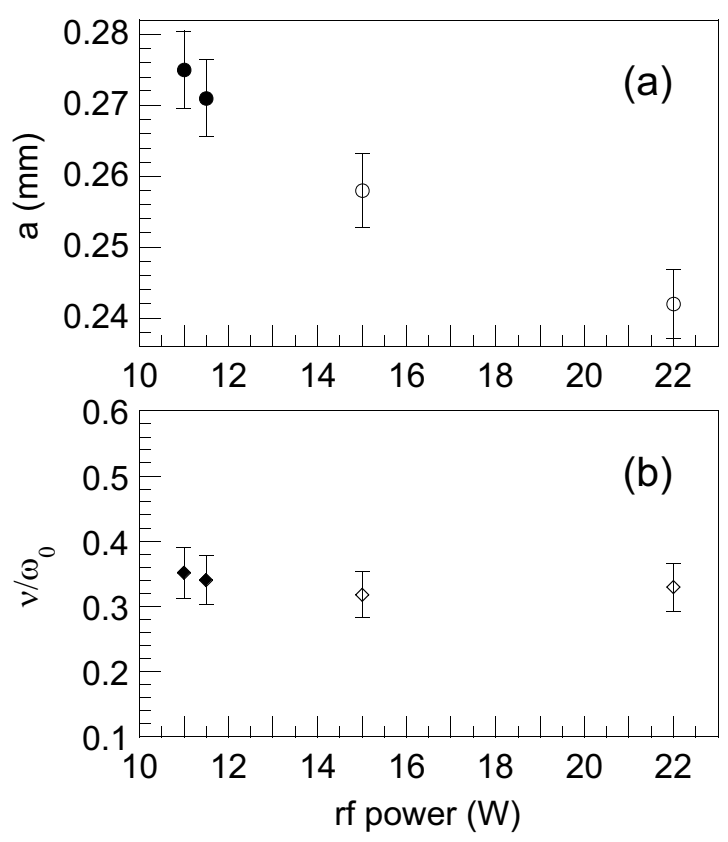

FIG. 1. (a) Mean interparticle spacing $a$ and (b) normalized neutral gas damping rate $\nu / \omega_{0}$ as functions of the discharge power $P_{\text {rf }}$. Solid symbols indicate experimental runs where MCI occurred. The argon pressure was $p_{\mathrm{Ar}}=12 \mathrm{~Pa}$.

and $\left.P_{\mathrm{rf}}\right)$ are different from theoretical ones $\left(\nu / \omega_{0}\right.$ and the vertical confinement parameter $\Omega_{z}$ ). Turning either of the two experimental "knobs" will change both theoretical control parameters, sometimes in a complicated way. This is in part due to the self-adjustable levitation height of the particle suspension, which therefore senses 

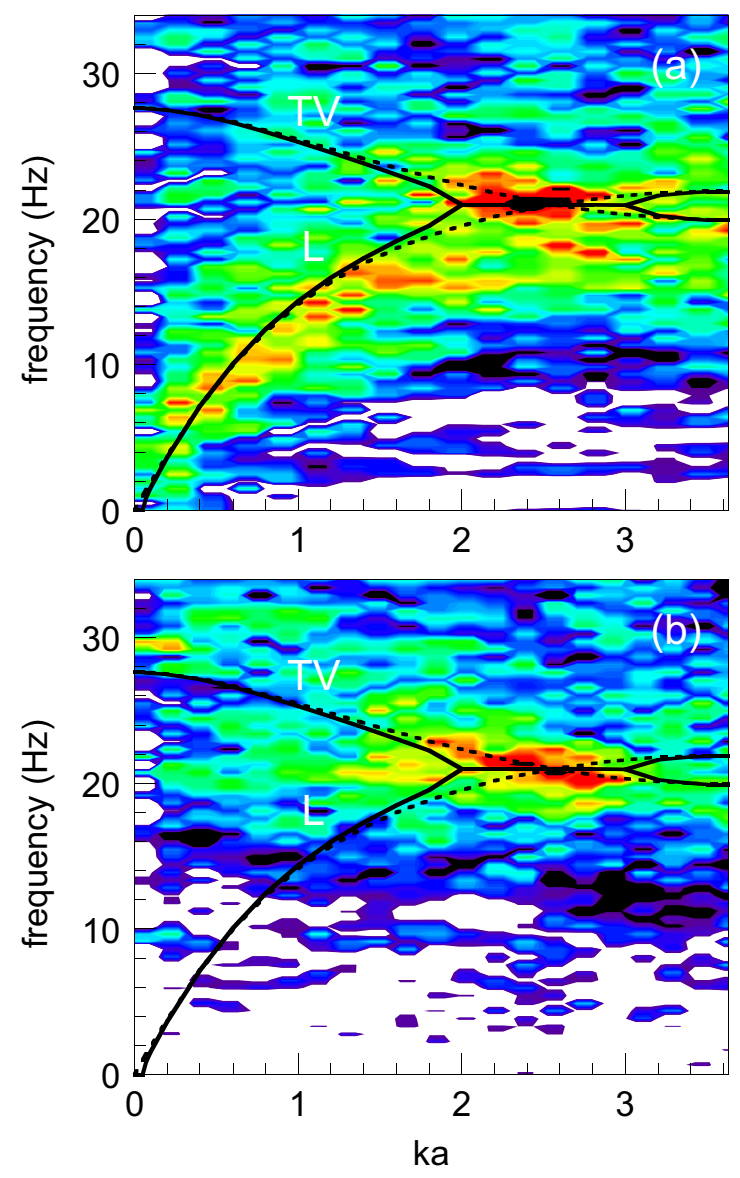

FIG. 2. Fluctuation spectra of the particle velocity: (a) longitudinal in-plane mode and (b) transverse out-of-plane mode. Spectral intensity is shown in arbitrary units and with $\log _{10}$ scale. The wave propagation direction was $\theta \simeq 0^{\circ}$ (see the text for more details). The first Brillouin zone for $\theta=0^{\circ}$ is shown; wave number $k$ is normalized by the inverse interparticle spacing $a^{-1}$. The solid lines are theoretical dispersion relations of the coupled L and TV modes [see Eq.(6)], the dashed lines are for the uncoupled modes [see Eq.(5)]. The spectra are for the experimental run \#7 in Table I.

different parts of the plasma sheath with different plasma conditions. A combination of the higher levitation height due to the sheath expansion at lower $P_{\mathrm{rf}}$ and a modified horizontal confinement apparently leads to the larger interparticle spacing $a$.

At the very onset of MCI, we observed a steady-state regime where MCI was present but its growth was suppressed by the neutral gas friction (experimental run \#7 in Table I). Suppressed MCI caused heating up of the plasma crystal but did not lead to its melting. Such a regime was theoretically predicted and observed in a molecular-dynamics simulation in Ref. ${ }^{3}$. Fig. 2 shows the particle velocity fluctuation spectra of the longitudinal in-plane and transverse out-of-plane modes. Both spectra contain "hot spots" at their intersection which are indicative of deep mode crossing. Fig. 3(a) shows fluctuation spectrum of the particle velocity (longitudinal
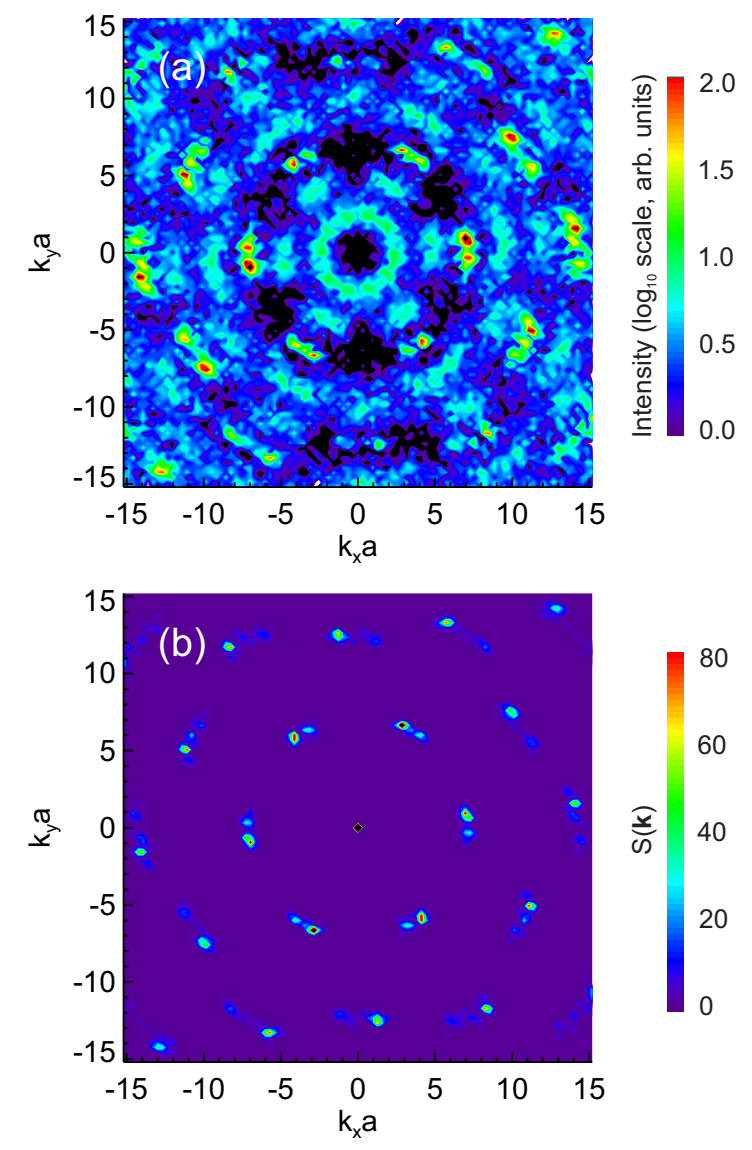

FIG. 3. (a) Fluctuation spectrum of the particle velocity (longitudinal in-plane mode) in the $\mathbf{k}$-plane (integrated over frequency in the range of $20 \leq|f| \leq 22 \mathrm{~Hz}$ ). The circular feature at $k a \simeq 2.4$ is characteristic of the deep mode crossing ${ }^{3}$. (b) Static structure factor $S(\mathbf{k})$ of the particle suspension. A highly-ordered crystal structure is evident despite deep mode crossing. Wave number is normalized by the inverse interparticle spacing $a^{-1}$. Experimental parameters were as in Fig. 2.

in-plane mode) integrated over the hot-spot frequency. The circular feature at $k a \simeq 2.4$ in this figure is characteristic of the deep mode crossing ${ }^{3}$. Figs. 2, 3(a) clearly establish the occurrence of MCI in this experimental run. Yet the plasma crystal remained ordered, as indicated by its static structure factor $S(\mathbf{k})$ in Fig. 3(b). Since in this steady-state regime the crystal did not melt, this regime is particularly convenient for studying various features of the MCI such as angular dependence and damping threshold.

\section{THEORETICAL MODEL}

We analyze our experimental observations in the framework of a relatively simple theoretical model which is described below. The 2D plasma crystal is modelled as a suspension of identical particles in a parabolic vertical confinement provided by the balance of the plasma sheath's electrostatic force and gravity. Much weaker 
horizontal confinement, which defines the finite diameter of the particle suspension in experiments, is ignored in the model. The particles are arranged in a triangular lattice with hexagonal symmetry, i.e., their equilibrium positions are located at

$$
\mathbf{r}_{n}^{(0)}=\left\{x_{n}^{(0)}, y_{n}^{(0)}\right\} \equiv\left\{\frac{\sqrt{3}}{2} \alpha a,\left(\beta+\frac{1}{2} \alpha\right) a\right\}
$$

where the pairs of integer numbers $n=\{\alpha, \beta\}$ label the lattice sites and $a$ is the crystal constant. The plasma wakes created by the positive ions streaming past the negatively charged particles are modelled as point-like positive charges $q$ located at a distance $\delta$ downstream of the ion flow beneath each particle $e^{27,28}$.

\section{A. Coupled wave modes}

Coupled longitudinal in-plane (L) and transverse outof-plane (TV) waves naturally excited and sustained in a $2 \mathrm{D}$ plasma crystal can be described by the following equations $^{29}$ :

$$
\begin{aligned}
& \ddot{\widetilde{x}}+\nu \dot{\widetilde{x}}=-\Omega_{L}^{2} \widetilde{x}+i \Omega_{W}^{2} \widetilde{z}+\widetilde{a}_{x}, \\
& \ddot{\widetilde{z}}+\nu \dot{\widetilde{z}}=-\Omega_{V}^{2} \widetilde{z}+i \Omega_{W}^{2} \widetilde{x}+\widetilde{a}_{z},
\end{aligned}
$$

where $\widetilde{x}$ and $\widetilde{z}$ are the complex amplitudes of the $\mathrm{L}$ and TV waves, respectively, $\nu$ is the neutral gas (Epstein) damping rate, and $\widetilde{\mathbf{a}}=\left\{\widetilde{a}_{x}, \widetilde{a}_{z}\right\}$ are time-dependent random accelerations defined by zero mean and an isotropic time-independent variance:

$$
\begin{array}{r}
\left\langle\widetilde{a}_{x}\right\rangle=\left\langle\widetilde{a}_{z}\right\rangle=0, \\
\left\langle\widetilde{a}_{x} \widetilde{a}_{z}^{*}\right\rangle=0, \\
\left\langle\left|\widetilde{a}_{x}\right|^{2}\right\rangle=\left\langle\left|\widetilde{a}_{z}\right|^{2}\right\rangle=\text { const. }
\end{array}
$$

These accelerations define the amplitudes of the naturally excited waves ${ }^{29}$. $\Omega_{L, V}$ are the eigenfrequencies of the $\mathrm{L}$ and TV modes, whereas $\Omega_{W}$ is the wake coupling frequency; their functional dependencies on the screening parameter $\kappa$ and the wave number $k$ are given below, see Eq. 10.

Many important conclusions can be done even without the detailed knowledge of these functional dependencies. In particular, the dispersion relation of the eigenwaves in the form $\widetilde{x}, \widetilde{z} \propto \exp \left(i k x^{(0)}-i \omega t\right)$ is given by

$$
\left[\Omega^{2}-\Omega_{V}^{2}\right]\left[\Omega^{2}-\Omega_{L}^{2}\right]+\Omega_{W}^{4}=0, \Omega^{2} \equiv \omega(\omega+i \nu) .
$$

In the absence of plasma wakes $\left(\Omega_{W} \equiv 0\right)$, the L and TV eigenwaves are fully decoupled, i.e.,

$$
\begin{gathered}
\omega_{1}=-i \nu / 2 \pm \sqrt{\Omega_{L}^{2}-\nu^{2} / 4}, \\
\omega_{2}=-i \nu / 2 \pm \sqrt{\Omega_{V}^{2}-\nu^{2} / 4}
\end{gathered}
$$

and can propagate $\left[\operatorname{Re}\left(\omega_{1,2}\right) \neq 0\right]$ if and only if $\Omega_{L, V}>$ $\nu / 2$. This is a rather weak restriction, which is in fact satisfied in all known plasma crystal experiments.
When the plasma wakes are taken into consideration $\left(\Omega_{W} \neq 0\right)$, the wave modes become coupled and their polarization mixes in-plane and out-of-plane oscillations:

$$
\begin{array}{r}
\left(\omega_{1,2}+i \nu / 2\right)^{2}= \\
\frac{1}{2}\left[\Omega_{V}^{2}+\Omega_{L}^{2}-\nu^{2} / 2 \pm\left(\Omega_{V}^{2}-\Omega_{L}^{2}\right) \sqrt{1-p}\right]
\end{array}
$$

where

$$
p=\frac{4 \Omega_{W}^{4}}{\left(\Omega_{V}^{2}-\Omega_{L}^{2}\right)^{2}}
$$

is a coupling parameter, a convenient measure of the mixed mode polarization ${ }^{29}$. For $p=0$, the $\mathrm{L}$ and TV modes are decoupled, the power of the mode coupling and mixed polarization increases rapidly with $p$ for $0<p<1$, while for $p=1$ the hybrid mode appears. It becomes a prominent feature of the wave pattern for $p>1$. It is important that the coupling parameter strongly depends on the lattice parameters and the wave number while it does not explicitly depend on the friction rate.

In the case of $p<1$, the wave modes are fully separated, stable, and their damping rate is always non-zero and wave-number independent, $\operatorname{Im}\left(\omega_{1,2}\right)=-\nu / 2$. They have to be externally supported, e.g., as naturally excited waves. For $p>1$, the hybrid mode appears first at short wavelengths; its $k$-space localization is restricted by the constraint $p(k) \geq 1$. The hybrid waves are also damped. Their damping rate is wave-number dependent. At lower discharge pressures, when the damping rate is low, the hybrid mode destabilizes the monolayer, the mode coupling instability develops, and the crystal layer melts ${ }^{3,4}$. At relatively high damping rate, there is a unique opportunity to observe undamped hybrid waves.

\section{B. Suppressed $\mathrm{MCl}$ : critical damping rate}

Corresponding stabilization criterion is formulated as follows. Note that the equation (4) could be equivalently rewritten as:

$$
\begin{array}{r}
{\left[\xi-\Omega_{V}^{2}\right]\left[\xi-\Omega_{L}^{2}\right]+2 i \nu\left[\xi-\left\langle\Omega^{2}\right\rangle\right]+\Omega_{W}^{4}-\nu^{2} \xi=0,} \\
\xi \equiv \omega^{2},\left\langle\Omega^{2}\right\rangle=\frac{\Omega_{V}^{2}+\Omega_{L}^{2}}{2}(8)
\end{array}
$$

At the instability threshold, $\omega$ must be real. Therefore, separately equating to zero the real and imaginary parts of the LHS of equation (8), one gets the instability threshold as a constraint:

$$
\begin{array}{r}
\omega^{2}=\omega_{\mathrm{hs}}^{2} \equiv \frac{\Omega_{V}^{2}+\Omega_{L}^{2}}{2}, \\
\nu^{2}=\nu_{\mathrm{cr}}^{2} \equiv \frac{\Omega_{W}^{4}-\left[\omega_{\mathrm{hs}}^{2}-\Omega_{L}^{2}\right]^{2}}{\omega_{\mathrm{hs}}^{2}},
\end{array}
$$

where $\omega_{\text {hs }}$ is the hot spot frequency. Since $\operatorname{Im}(\omega) \equiv 0$ for this mode, it is neutrally stable $e^{30}$. Moreover, as is easy to check, the imaginary parts of all other spectral 
components remain negative in these conditions. Hence, the suppressed MCI mode must be the brightest part of the wave spectrum, in an excellent agreement with our observations, see Fig. $2^{31}$. It is worth noting that the appearance of a hot spot in the wave spectrum does not necessary mean melting of the lattice layer ${ }^{3}$. Indeed, the plasma crystal remained ordered in the conditions of Fig. 2, as evidenced by its static structure factor $S(\mathbf{k})$ shown in Fig. 3(b), even though a deep crossing of the L and TV modes occurred.

\section{Dispersion relation parameters}

Generally, the parameters $\Omega_{L, V, W}$ are quite complicated functions of the wave propagation direction and wave number $3,4,29$. For our purpose, a rather simple null$\delta$ model $^{4}$ will work well. Moreover, to simplify description, we choose the "most unstable" wave propagation direction ${ }^{3}$, i.e., $\theta=0$, corresponding to the spectra in Fig. 2. $\Omega_{L, V, W}$ are then given by the following expressions:

$$
\begin{aligned}
\Omega_{L}^{2} & =2 \Omega_{0}^{2} S_{L}(k, \kappa), \\
\Omega_{V}^{2} & =\Omega_{z}^{2}-2 \Omega_{0}^{2} S_{V}(k, \kappa), \\
\Omega_{W}^{2} & =\Omega_{0}^{2} W S_{W}(k, \kappa),
\end{aligned}
$$

where $\Omega_{0}=\widetilde{Q}\left(4 \pi \epsilon_{0} m \lambda^{3}\right)^{-1 / 2}$ is the dust lattice frequency. The effective particle charge is introduced by $\widetilde{Q}^{2}=Q^{2}(1-\widetilde{q})$, where $\widetilde{q}=|q / Q|<1$ is the normalized wake charge. The dimensionless wake parameter is defined as

$$
W=\frac{\widetilde{q} \widetilde{\delta}}{1-\widetilde{q}}
$$

where $\widetilde{\delta}=\delta / a$. The sums $S_{L, V, W}(k, \kappa)$ run over all lattice sites $n=\{\alpha, \beta\}$, where $\alpha, \beta=(-\infty,+\infty)$, except $n=$ $\{0,0\}$ :

$$
\begin{aligned}
S_{L} & =\sum_{n}\left[\frac{r_{x}^{2}}{r^{2}} \Xi\left(\frac{r}{\lambda}\right)-\Lambda\left(\frac{r}{\lambda}\right)\right] \sin ^{2}\left(k r_{x} / 2\right), \\
S_{V} & =\sum_{n} \Lambda\left(\frac{r}{\lambda}\right) \sin ^{2}\left(k r_{x} / 2\right), \\
S_{W} & =\sum_{n} \Xi\left(\frac{r}{\lambda}\right) \frac{a r_{x}}{r^{2}} \sin \left(k r_{x}\right),
\end{aligned}
$$

where $r_{x}=\sqrt{3} a \alpha / 2, r_{y}=a(\beta+\alpha / 2), r=\sqrt{r_{x}^{2}+r_{y}^{2}}$, $\kappa=a / \lambda, \Xi(x)=\frac{3+3 x+x^{2}}{x^{3}} e^{-x}$ and $\Lambda(x)=\frac{1+x}{x^{3}} e^{-x}$.

An apparent advantage of the model (10-12) is that it is completely defined by only three parameters $\kappa=a / \lambda$, $\Omega_{0}$, and $W$, which can be extracted from the experimental data. This can be used as a diagnostic tool. The first two parameters are easy to calculate using a well known method ${ }^{26}$, where the speeds of longitudinal $\left(C_{L}\right)$ and transverse in-plane $\left(C_{T}\right)$ sound waves are measured
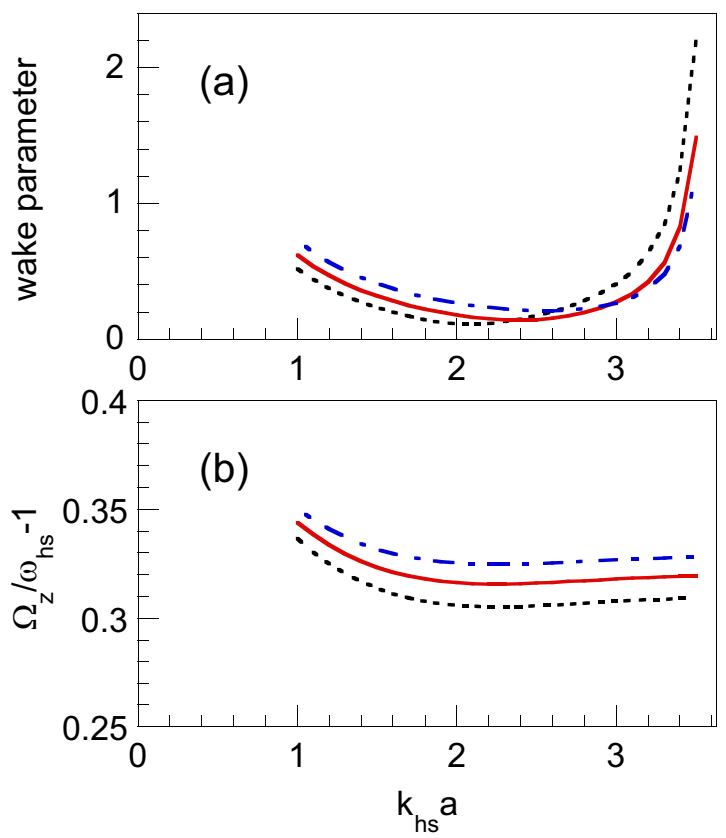

FIG. 4. Theoretical (a) wake parameter $W=\widetilde{q} \widetilde{\delta} /(1-\widetilde{q})$ and (b) normalized confinement parameter $\Omega_{z} / \omega_{\mathrm{hs}}-1$ as functions of the normalized hot spot wave number $k_{\mathrm{hs}} a$, see Eq. (14). The dotted curves are for $\omega_{\mathrm{hs}} / 2 \pi=20 \mathrm{~Hz}$, solid curves for $21 \mathrm{~Hz}$, and dash-dotted curves for $22 \mathrm{~Hz}$.

from the experimental spectra and compared with their analytical expressions:

$$
C_{\{L, T\}}^{2}=\frac{1}{2} \Omega_{0}^{2} \sum_{n}\left[\frac{r_{\{x, y\}}^{2}}{r^{2}} \Xi\left(\frac{r}{\lambda}\right)-\Lambda\left(\frac{r}{\lambda}\right)\right] r_{x}^{2} .
$$

The wake parameter $W$ can be readily obtained by plugging the measured MCI-induced hot spot position into equations (9). Apparently, the hot spot position is better measurable in the experimental spectra (see Fig. 2) than, e.g., the vertical confinement parameter $\Omega_{z}{ }^{32}$. Therefore, it is worth to consider, instead of Eq. (9), an equivalent inverse problem:

$$
\begin{gathered}
\Omega_{z}^{2}=2 \omega_{\mathrm{hs}}^{2}-\Omega_{L}^{2}+2 \Omega_{0}^{2} S_{V} \equiv F_{1}\left(k_{\mathrm{hs}}\right), \\
W^{2}=\frac{\nu_{\mathrm{cr}}^{2} \omega_{\mathrm{hs}}^{2}+\left[\omega_{\mathrm{hs}}^{2}-\Omega_{L}^{2}\right]^{2}}{\Omega_{0}^{4} S_{W}^{2}} \equiv F_{2}\left(k_{\mathrm{hs}}\right),
\end{gathered}
$$

where $k_{\mathrm{hs}}$ is the hot spot wave number. These expressions immediately give $\Omega_{z}$ and $W$ for the measured value of $k_{\mathrm{hs}}$, see Fig. 4.

We used the method described above to analyse the wave spectra of the crystal with suppressed MCI, see Fig. 2. The following results were obtained: $Q=9200 e$, $\kappa=0.76, \omega_{\mathrm{hs}} / 2 \pi=21 \mathrm{~Hz}, k_{\mathrm{hs}} a=2.4, \Omega_{z} / 2 \pi=27.6 \mathrm{~Hz}$, $W=0.14$. The measurement of the wake parameter $W$ is of particular interest since the knowledge of $W$ is crucial to predicting the behaviour of the plasma crystal in various experimental conditions. We are not aware of any prior experimental measurement of the wake parameter. Theoretical dispersion relations calculated using 
these parameters are shown as lines in Fig. 2. There is a good overall agreement between the experiment and theory. Our results show that the mode-coupling instability in 2D complex plasma crystals can be experimentally studied at relatively high gas pressures. Such experiments reveal interesting phenomena which can be successfully analysed despite relatively high neutral gas damping rate.

\section{ACKNOWLEDGMENTS}

The authors thank Mierk Schwabe for carefully reading the manuscript and for helpful comments. T.W.H. gratefully acknowledges support from grants NSF 1740203 and NASA 1571701. V.N. gratefully acknowledges the hospitality experienced at Baylor University.

${ }^{1}$ A. V. Ivlev and G. Morfill, Phys. Rev. E 63, 016409 (2000).

${ }^{2}$ L. Couëdel, V. Nosenko, A. V. Ivlev, S. K. Zhdanov, H. M. Thomas, and G. E. Morfill, Phys. Rev. Lett. 104, 195001 (2010).

${ }^{3}$ L. Couëdel, S. K. Zhdanov, A. V. Ivlev, V. Nosenko, H. M. Thomas, and G. E. Morfill, Phys. Plasmas 18, 083707 (2011).

${ }^{4}$ S. K. Zhdanov, A. V. Ivlev, and G. E. Morfill, Phys. Plasmas 16, 083706 (2009).

${ }^{5}$ A. Ivlev, H. Löwen, G. Morfill, C. P. Royall, Complex Plasmas and Colloidal Dispersions: Particle-resolved Studies of Classical Liquids and Solids, Series in Soft Condensed Matter Vol. 5 (World Scientific, Singapore, 2012).

${ }^{6}$ H. M. Thomas and G. E. Morfill, Nature (London) 379, 806 (1996).

${ }^{7}$ V. Nosenko, S. K. Zhdanov, A. V. Ivlev, C. A. Knapek, and G. E. Morfill, Phys. Rev. Lett. 103, 015001 (2009).

${ }^{8}$ A. Melzer, A. Schella, J. Schablinski, D. Block, and A. Piel, Phys. Rev. E 87, 033107 (2013).

${ }^{9}$ S. Nunomura, D. Samsonov, S. Zhdanov, and G. Morfill, Phys. Rev. Lett. 96, 015003 (2006).

${ }^{10}$ V. Nosenko and J. Goree, Phys. Rev. Lett. 93, 155004 (2004).

${ }^{11}$ A. Gavrikov, I. Shakhova, A. Ivanov, O. Petrov, N. Vorona, and V. Fortov, Phys. Lett. A 336, 378 (2005).

${ }^{12}$ P. Hartmann, M. C. Sándor, A. Kovács, and Z. Donkó, Phys. Rev. E 84, 016404 (2011).

${ }^{13}$ V. Nosenko, S. K. Zhdanov, A. V. Ivlev, G. E. Morfill, J. Goree, and A. Piel, Phys. Rev. Lett. 100, 025003 (2008).
${ }^{14}$ S. Nunomura, J. Goree, S. Hu, X. Wang, A. Bhattacharjee, K. Avinash, Phys. Rev. Lett. 89, 035001 (2002).

${ }^{15}$ A. Piel, V. Nosenko, J. Goree, Phys. Rev. Lett. 89, 085004 (2002).

${ }^{16}$ S. K. Zhdanov, S. Nunomura, D. Samsonov, and G. Morfill, Phys. Rev. E 68, 035401 (2003).

${ }^{17}$ K. Avinash, P. Zhu, V. Nosenko, and J. Goree, Phys. Rev. E 68, 046402 (2003).

${ }^{18}$ B. Liu, J. Goree, and V. Nosenko, Phys. Plasmas 10, 9 (2003).

${ }^{19} \omega_{0}=Q\left(4 \pi \epsilon_{0} m a^{3}\right)^{-1 / 2}$, where $Q$ and $m$ are the particle charge and mass, respectively, and $a$ is the mean interparticle spacing.

${ }^{20}$ V. Nosenko, S. K. Zhdanov, H. M. Thomas, J. Carmona-Reyes, and T. W. Hyde, Phys. Rev. E 96, 011201(R) (2017).

${ }^{21}$ V. Nosenko, S. K. Zhdanov, H. M. Thomas, J. Carmona-Reyes, and T. W. Hyde, EPL 112, 45003 (2015).

${ }^{22}$ C. M. Boessé, M. K. Henry, T. W. Hyde, and L. S. Matthews, Advances in Space Research 34, 2374 (2004).

${ }^{23}$ Marketed by Microparticles GmbH, see http://microparticles.de.

${ }^{24}$ C.-R. Du, V. Nosenko, S. Zhdanov, H. M. Thomas, and G. E. Morfill, Phys. Rev. E 89, 021101(R)(2014).

${ }^{25} \mathrm{U}$. Konopka (unpublished).

${ }^{26}$ S. Nunomura, J. Goree, S. Hu, X. Wang, A. Bhattacharjee, Phys. Rev. E 65, 066402 (2002).

${ }^{27}$ A. Melzer, V. A. Schweigert, I. V. Schweigert, A. Homann, S. Peters, and A. Piel, Phys. Rev. E 54, R46 (1996).

${ }^{28}$ M. Lampe, G. Joyce, G. Ganguli, and V. Gavrishchaka, Phys. Plasmas 7, 3851 (2000).

${ }^{29}$ J. K. Meyer, I. Laut, S. K. Zhdanov, V. Nosenko, H. M. Thomas, Phys. Rev. Lett. 119, 255001 (2017).

${ }^{30}$ The spectral energy density of this mode is therefore formally unlimited. This means that other saturation mechanisms should be taken into account, e.g., phonon diffusion in the $k$-space, which very effectively removes energy from the hot $\operatorname{spots}^{33}$.

${ }^{31}$ For any particular value of the damping rate, e.g., $\nu=\nu_{\mathrm{cr}}$, the fourth order equation (8) has four solutions. Two more solutions, in addition to $\omega= \pm \omega_{\mathrm{hs}}$, are easy to find by rewriting (8) as follows:

$$
\left[\omega^{2}-\omega_{\mathrm{hs}}^{2}\right]\left[(\omega+i \nu)^{2}-\omega_{\mathrm{hs}}^{2}\right]=\omega_{\mathrm{hs}}^{2}\left[\nu^{2}-\nu_{\mathrm{cr}}^{2}\right],
$$

where the critical parameters are as defined in Eq. (9). It immediately gives us two additional, now damped, solutions:

$$
\nu=\nu_{\mathrm{cr}}, \omega=-i \nu \pm \omega_{\mathrm{hs}} .
$$

${ }^{32}$ The vertical confinement parameter can be formally defined as the long-wavelength limit of the out-of-plane branch, $\Omega_{z}=$ $\lim _{k \rightarrow 0} \Omega_{V}$.

${ }^{33}$ I. Laut, S. K. Zhdanov, C. R. Räth, H. M. Thomas, and G. E. Morfill, Phys. Rev. E 93, 013204 (2016). 